\title{
Machine-learned analysis of global and glial/opioid intersection-related DNA methylation in patients with persistent pain after breast cancer surgery
}

\author{
Dario Kringel ${ }^{1}$, Mari A. Kaunisto ${ }^{4}$, Eija Kalso ${ }^{2}$ and Jörn Lötsch ${ }^{1,3^{*}}$ (1)
}

\begin{abstract}
Background: Glial cells in the central nervous system play a key role in neuroinflammation and subsequent central sensitization to pain. They are therefore involved in the development of persistent pain. One of the main sites of interaction of the immune system with persistent pain has been identified as neuro-immune crosstalk at the glialopioid interface. The present study examined a potential association between the DNA methylation of two key players of glial/opioid intersection and persistent postoperative pain.

Methods: In a cohort of 140 women who had undergone breast cancer surgery, and were assigned based on a 3year follow-up to either a persistent or non-persistent pain phenotype, the role of epigenetic regulation of key players in the glial-opioid interface was assessed. The methylation of genes coding for the Toll-like receptor 4 (TLR4) as a major mediator of glial contributions to persistent pain or for the $\mu$-opioid receptor (OPRM1) was analyzed and its association with the pain phenotype was compared with that conferred by global genome-wide DNA methylation assessed via quantification of the methylation in the retrotransposon LINE1.

Results: Training of machine learning algorithms indicated that the global DNA methylation provided a similar diagnostic accuracy for persistent pain as previously established non-genetic predictors. However, the diagnosis can be based on a single DNA based marker. By contrast, the methylation of TLR4 or OPRM1 genes could not contribute further to the allocation of the patients to the pain-related phenotype groups.

Conclusions: While clearly supporting a predictive utility of epigenetic testing, the present analysis cannot provide support for specific epigenetic modulation of persistent postoperative pain via methylation of two key genes of the glial-opioid interface.
\end{abstract}

\section{Introduction}

Persistent pain is a major healthcare problem $[1,2]$, currently regarded as resulting from neural plasticity including peripheral [3] and central sensitization $[4,5]$. The interaction between neurons and glial cells (e.g., microglia and astrocytes) is critical for the initiation and maintenance of persistent pain [6]. Increasing evidence

\footnotetext{
* Correspondence: j.loetsch@em.uni-frankfurt.de

${ }^{1}$ Institute of Clinical Pharmacology, Goethe-University, Theodor-Stern-Kai 7, 60590 Frankfurt am Main, Germany

${ }^{3}$ Fraunhofer Institute of Molecular Biology and Applied Ecology-Project

Group Translational Medicine and Pharmacology (IME-TMP),

Theodor-Stern-Kai 7, 60590 Frankfurt am Main, Germany

Full list of author information is available at the end of the article
}

suggests that activation of glial cells contributes to the pathogenesis of persistent pain via neuron-glial interactions $[7,8]$. The pro-inflammatory effects of the activation of Toll-like receptors (TLR), positioned at the neuroimmune interface on glia cells, sensory neurons, and other cell types can enhance nociceptive processing leading to exaggerated and unresolved pain [9]. This is mediated in particular by TLR4 that has been shown to induce microglial activation and cytokine production [10]. Moreover, the TLR4 inhibitor (+)-naloxone was able to reverse established neuropathic pain in a nerve injury induced in rats [11].

While (+)-naloxone is inactive at opioid receptors, one of the main sites of interaction of the immune system

(c) The Author(s). 2019 Open Access This article is distributed under the terms of the Creative Commons Attribution 4.0 International License (http://creativecommons.org/licenses/by/4.0/), which permits unrestricted use, distribution, and 
with persistent pain has been identified as neuroimmune crosstalk at the glial-opioid interface [12, 13]. Indeed, glial cells are involved in opioid actions [14, 15]. For example, the putative toll-like receptor 4 antagonist ibudilast restored morphine-induced antinociception in morphine-tolerant rats [16]. Similarly, minocycline attenuated morphine tolerance in mouse models of neuropathic pain by inhibiting microglial activation [14]. This particular effect on morphine action renders the $\mu$ opioid receptor a key player in glia-opioid crosstalk since the vast majority of current opioid analgesics are mainly $\mu$-opioid receptor agonists.

The involvement of neuroimmune processes in persistent pain is under genetic control [17], suggesting that it may also be under epigenetic control. Indeed, classical epigenetic mechanisms including changes in DNA methylation and histone modifications have been shown to contribute to the development and treatment responsiveness of persistent pain [18]. This was seen at both single gene and global DNA methylation levels. For example, the methylation level of the mu-opioid receptor gene (OPRM1) has been associated with acute and chronic postsurgical pain [19]. Similarly, different methylation levels of LINE1, which is a retrotransposon of viral provenience spread in approximately half million copies across the human genome [20-22] and therefore used as a marker of global DNA methylation, correlated with different intensity scores of persistent pain [23].

In the present analysis, the methylation status of TLR4, OPRM1, and LINE1 was assessed for its association with the persistence of postsurgical pain. DNA samples and pain data were available from a cohort of 1000 women who had undergone breast cancer surgery [24], among whom $n=70$ patients had developed persistent postsurgery pain, based on ratings acquired up to 36 months after surgery [25]. The previous analysis of the same samples had focused on a role of genetic variants in a selection of pain-relevant genes including the two selected for the present analysis. Indeed, 21 variants in 13 different genes were found to be relevant to the assignment of a patient to either the persistent pain or the non-persistent pain phenotype group [26]. OPRM1 variants but not TLR4 variants had been among the relevant genetic markers. Considering that in addition to nucleotide sequence changes, the methylation status can also include the expression of genes, the focus on the glial/ opioid intersection in persistent pain was further pursued in the present epigenetic assessments.

\section{Methods}

\section{Patients and pain phenotype}

The study followed the Declaration of Helsinki, and both the Coordinating Ethics Committee (journal number 136/ E6/2006) and the Ethics Committee of the Department of
Surgery (148/E6/05) of the Hospital District of Helsinki and Uusimaa approved the study protocol. Informed written consent was obtained from each patient. The cohort has been described in detail previously [24, 27]. In brief, 1000 women aged 28-75 years suffering from unilateral non-metastasized breast cancer were enrolled during the preoperative visit. They were treated with breastconserving surgery or mastectomy, sentinel node biopsy, and/or axillary clearance. Exclusion criteria were neoadjuvant therapy [28] and immediate breast reconstruction surgery. Perioperative analgesia was standardized consisting of preoperative oral acetaminophen, perioperative remifentanil, and postoperative intravenous oxycodone during the first 20 postoperative hours, and ibuprofen or a combination of acetaminophen and codeine during the first postoperative week; no regional anesthesia was used. Adjuvant treatments were given according to international guidelines [27].

As reported previously $[25,29,30]$, post-surgical pain intensity was assessed at months 1, 6, 12, 24, and 36 after surgery using numerical rating scale (NRS) ranging from 0 (no pain) to 10 (the most severe pain that can be imagined) [31]. For the diagnosis of persistent pain, NRS data acquired 12-36 months after the surgery were used. As discussed previously [32], due to ongoing adjuvant therapies, this can be considered as more adequately reflecting the clinical setting of breast cancer surgery than the original definition of persistent post-surgical pain, which proposes a cut-off at 2 months [33]. Persistent pain was defined on the basis of NRS ratings as described previously [25], i.e., patients were assigned to the "persistent pain" subgroup if the following conditions applied: NRSmonth36> 3 and NRSmonth12. month $36>0$ and (NRSmonth36NRSmonth24) $\geq 0$, whereas patients were assigned to the "non-persistent pain" group if NRSmonth $36 \leq 3$ and NRSmonth12. month $36 \leq 3$. Applying these criteria to the cohort of 1000 women led to the diagnosis of persistent pain in $n=70$ patients [25]. For comparison, a similarly sized age and body mass index (BMI)-matched subsample was drawn from the patients who had not developed persistent postsurgical pain.

\section{Quantification of DNA methylation}

DNA methylation levels of CpG sites in OPRM1, TLR4, and LINE1 were quantified by means of Pyrosequencing ${ }^{\mathrm{TM}}$ assays as used in previous assessments of epigenetic influences on pain [23, 34, 35]. In brief, genomic DNA was extracted from $200 \mu \mathrm{l}$ of full blood on a BioRobot EZ1 workstation applying the blood and body fluid spin protocol provided in the EZ1 DNA Blood $200 \mu$ l Kit (Qiagen, Hilden, Germany) and eluted in a final concentration of 50 $\mathrm{ng} / \mu \mathrm{l}$. Methylation levels were quantified using Pyrosequencing $^{\text {TM }}$ assays described elsewhere in full detail [36-39]. The assays were designed to examine the methylation 
status at (i) four CpG sites in the promoter region of LINE1 at bp position - 605, - 593, - 590, and - 583; (ii) six CpG sites in the promoter region of OPRM1 at bp position - 60, - 50, - 32, - 25, - 18, and - 14; and (iii) four CpG sites in the promoter region of TLR4 at bp position $-75,-67,-58$, and -51 , all relative to the start codon. PCR reactions were run on a Mastercycler nexus gradient flexlid device (Eppendorf, Hamburg, Germany) in a 50- $\mu$ l reaction volume including 5- $\mu$ l bisulfite-treated DNA, mixed with $0.5 \mu \mathrm{l}$ $\mathrm{MyTaq}^{\mathrm{ma}}$ HS DNA polymerase $(5 \mathrm{U} / \mu \mathrm{l})$ (Bioline, Luckenwalde, Germany), $10 \mu \mathrm{l} 5 \times$ MyTaq reaction buffer, $0.2 \mu \mathrm{l}$ of each PCR primer $(100 \mu \mathrm{M})$, and $34.1 \mu \mathrm{l}$ HPLC-purified water.

Pyrosquencing $^{\text {Tu }}$ (Qiagen, Hilden, Germany) took place as described previously [23]. In brief, $50 \mu \mathrm{l}$ of the PCR templates were processed and purified with the PyroMark Vacuum Prep Worktable (Biotage, Uppsala Sweden) and subsequently annealed to the sequencing primer at $80{ }^{\circ} \mathrm{C}$ for $2 \mathrm{~min}$ as instructed by the manufacturer. Sequence analysis took place on a PSQ 96 MA System using the PyroMark Gold Q96 Reagents (Qiagen, Hilden, Germany). Pyro Q-CpG methylation software (version 1.0.9) had been used to determine the nucleotide dispensation order. The methylation values represent the mean percentage methylation across all CpG sites, which were measured in duplicate samples within one run. In addition, each sample was measured in two independent runs, which were subsequently averaged. To verify the accuracy of the analysis, each run included control DNA from the EpiTect PCR Control DNA Set (Qiagen, Hilden, Germany) that contained both bisulfite converted $100 \%$ methylated, as well as unmethylated, DNA as positive controls and unconverted unmethylated DNA as a negative control.

\section{Data analysis}

Data analysis was performed using the $\mathrm{R}$ software package (version 3.4.4 for Linux; http://CRAN.R-project.org/ [40]) on an Intel Core $19^{\circ}$ computer running on Ubuntu Linux 18.04.1 64-bit). Epigenetic data comprised methylation status, measured in percentage, of $d=6 \mathrm{CpG}$ sites in the OPRM1 gene, $d=4$, CpG sites in the TLR4 gene, and $d=4$ CpG sites in the LINE1 retrotransposon, acquired in $n=70 \mathrm{pa}-$ tients with persistent pain and $n=70$ patients assigned to the "non-persistent pain" subgroup. From this $14 \times 140$-sized data matrix, 15 single values $(0.76$ $\%)$ were missing. Following exploration of the data distribution, which indicated that no transformation was needed, and following a negative test for possible outliers (Grubbs test [41]), gaps in the data space were closed by means of $\mathrm{k}$-nearest neighbors imputation of the missing values. This was done using the $\mathrm{R}$ libraries "outliers" (https://cran.r-project.org/package=outliers [42]) and "DMwR" (https://cran.r-project.org/package= DMwR [43]).

The data analysis aimed to identify (i) whether there is an association between the DNA methylation and the persistence of pain, and (ii) which of the assessed genes was implicated in this association. The approach was data-driven and focused on the information about the pain phenotype conferred by the methylation status of the two selected genes or the global methylation status. Specifically, the data analysis followed three main steps. In the first step, basic statistical assessments were performed including assessment of differences in DNA methylation between the two pain phenotype groups, which was done by means of Wilcoxon tests and applying a correction for multiple testing according to Bonferroni [44]. In addition, Spearman correlations [45] were calculated among the components of the matrix of $\mathrm{CpG}$ sites and of the original NRS ratings acquired at 1, 6, 12, 24 , and 36 months after the surgery. The second step addressed the emergence of structures in the data space of DNA methylation patterns that reflected the known pain phenotype group structure of the patients. The third step of the analysis aimed at associating the methylation status of particular genes with the membership to the pain phenotype groups.

\section{Methylation pattern analysis using data structure detection}

The data space was explored for structures in the DNA methylation patterns that coincided with the known pain phenotype group structure. Unsupervised machinelearning was employed for data structure detection. Specifically, a parameter-free focusing projection method of a polar swarm, Pswarm, was used that exploits concepts of self-organization and swarm intelligence. It uses a swarm of intelligent agents called DataBots, which are self-organizing artificial "life forms" that carry vectors of the data. The data space was explored for distance-based structures. Following successful swarm learning on methylation data, rescaled into the range $[0, \ldots, 100]$, DataBots carrying items with similar features were placed close to each other in groups on the projection grid. The identification of emergent structures in the learned structure was further enhanced by calculating the distances between data points using the so-called Umatrix $[46,47]$. Every value (height) in the U-matrix depicts the average high-dimensional distance of a prototype in relation to all immediate neighboring prototypes regarding grid position. The corresponding visualization technique is a topographical map facilitating the recognition of data structures or clusters. These calculations were performed using the R library "DatabionicSwarm" (https://cran.r-project.org/package=DatabionicSwarm [48]). 
For internal validation, data structures were assessed again by means of principal component analysis (PCA) [49]. Specifically, a non-standard implementation of the PCA was used consisting of the "PC-corr" algorithm [50]. By automatically testing various data transformation and analyzing the associated group separations, using quantitative evaluations expressed as $p$ value, AUC, and AUPR, assessing any types of normalization and dimension, it permits to find the best results of a PCA. As it calculates various quality measures for every combination of PC, normalization, and centering, it allows the optimal selection of PC for data projection. This analysis was performed using an $\mathrm{R}$ script provided with the description of the PC-corr analysis (pccorrv2.R, https:// github.com/biomedical-cybernetics/PC-corr_net [50]).

\section{Association analysis of specific gene methylation sites with pain phenotype group membership}

Following the establishment of a data structure supporting a segregation of pain phenotype groups on the basis of the DNA methylation pattern, the contribution of particular genes or $\mathrm{CpG}$ sites to the group separation was assessed. Firstly, the results of the PCA performed in the previous analytical step were further explored. This was addressed by calculating the loadings of the CpG sites with the PCA components that explained relevant fractions of the total variance in the data.

Secondly, for internal validation, supervised machine learning methods were applied to narrow the focus on particular CpG sites respectively carrying genes. Specifically, supervised methods were implemented as (i) classification and regression trees [51], (ii) k-nearest neighbors [52], (iii) support vector machines [53], (iv) multinomial regression [54], and (v) naïve Bayesian classifiers [55]. Classification and regression trees use a tree data structure created with conditions on variables (parameters) as vertices and classes (diagnoses) as leaves.

Briefly, tree-structured rule-based classifiers [56] analyze ordered variables $x_{i}$, such as the present results of methylation analyses [scaled $0-100 \%$ ], by recursively splitting the data at each node into children nodes, starting at the root node. During learning, the splits are modified such that misclassification is minimized. The Gini impurity was used to find optimal (local) dichotomic decisions as used for the classification and regression tree method (CART) [51]. The calculations were done using the "rpart" function of the similarly named $\mathrm{R}$ package (B. Ripley; https://cran.r-project.org/package= rpart). The k-nearest neighbor (kNN) classification [52] provides a non-parametric method that belongs to the most frequently used algorithms in data science although it is one of the basic methods in machine learning. During kNN model building, the entire labeled training dataset is stored while a test case is placed in the feature space in the vicinity of the test cases at the smallest high-dimensional distance. The test case receives the class label according to the majority vote of the class labels of the $k$ training cases in its vicinity. The present analyses were performed in $k=5$ and the Euclidean distance as the default of the $\mathrm{R}$ package "KernelKnn" (Mouselimis L, https://cran.r-project.org/ package $=$ KernelKnn). Support vector machines are supervised learning methods that classify data mainly based on geometrical and statistical approaches employed for finding an optimum decision surface (hyperplane) that can separate the data points of one class from those belonging to another class in the highdimensional feature space [53]. Using a kernel function, the hyperplane is frequently selected in a way to obtain a trade-off between minimizing the misclassification rate and maximizing the distance of the plane to the nearest properly classified data point. In the present analysis, a Gaussian kernel with a radial basis was used. The analyses were done using the R library "kernlab" (https:// cran.r-project.org/package=kernlab [57]). Multinomial regression provides a method for estimating, from dichotomous or polychotomous data, the probability of occurrence of an event as a function of independent variables [54]. It employs sigmoid data transformation, such as the logit [58], to obtain a linearization, making the data accessible to techniques of multiple regression and its extensions, such as analysis of variance and covariance. The method extends logistic regression to the application on data in which the dependent variable may have a nominal scale with more than two levels, such as in the present three-class problem of three clinical olfactory diagnoses. The present implementation consisted of fitting multinomial log-linear models via neural networks as provided in the R library "nnet" (https://cran.rproject.org/package $=$ nnet [59]). Finally, naïve Bayesian classifiers were used that provide the probability that a data point being assigned to a specific class calculated by application of the Bayes' theorem [55]. The calculations were done using the $\mathrm{R}$ package "klaR" (https://cran.rproject.org/package $=\mathrm{klaR}[60]$ ).

The analyses were performed in cross-validation runs using 1000 times Monte Carlo [61] to obtain random splits of the original data set into training ( $2 / 3$ of the data) and test (1/3 of the data) data subsets. This was done using the R library "sampling" (https://cran.r-project.org/package $=$ sampling [62]). For all analyses, the data set was grouped proportionally, with respect to the two pain phenotypes, randomly split into a training data subset $(2 / 3$ of the patients) and a test data subset (the remaining $1 / 3$ of the patients). Training of the algorithms with methylation data was performed on the training data subset, and the trained algorithms were then used to identify the group membership of the cases 
belonging to the test data subset. As main test performance measure, the classification accuracy was used, i.e., the accuracy at which a patient was assigned to her correct pain phenotype group.

To comparatively evaluate the importance of the genes in this task, different combinations of gene-related CpG sites were used, comprising (i) the CpG sites in all three genes, (ii) in each gene separately, and (iii) in combinations of two genes each. Thus, supervised machine learning was used, mainly for knowledge discovery rather than to create a classifier, i.e., a biomarker, for prediction of persistent pain, for which the methylation status in only three genes was judged as unlikely to suffice. The underlying idea was that if an algorithm can be trained with methylation information to identify patients with persistent pain better than by guessing, the information is relevant for the clinical phenotype.

To avoid correct phenotype associations being a result of overfitting rather than based on methylation information, several measures against this weakness of machine learning algorithms were implemented. Firstly, prior to the data analysis, the classification algorithms were tuned with respect to available hyperparameters. For example, the number of $k$ in $\mathrm{kNN}$ was tested between 3 and 9 and the best performing variant was chosen. Secondly, analyses were performed in cross-validation runs using 1000 times Monte Carlo [61] resampling and data splitting into non-overlapping training and test data subsets. Thirdly, negative control data sets were created by random permutation of the methylation data in the respective training data subsets of each scenario. The expectation was that when trained with random and therefore meaningless data, the algorithm should not perform better than guessing when applied to the association of the pain phenotypes in the test data subsets. Fourthly, five different classifiers were applied to avoid the analysis relying on a single method in which occasional overfitting might have occurred.

\section{Results}

DNA methylation was quantified from $d=6,4$, or 4 CPG sites located in OPRM1, TLR4, and LINE1, respectively, in $n=70$ patients with persistent pain and in $n=$ 70 patients assigned to the "non-persistent pain" phenotype group based on the 3-year follow-up data after breast cancer surgery (Fig. 1a). The demographic data were the following: age $41-73$ years (median 58.5 years), BMI $18.4-36.2 \mathrm{~kg} / \mathrm{m}^{2}$, (median $25.6 \mathrm{~kg} / \mathrm{m}^{2}$ ) in the pain group; and age 40-73 years, (median 59 years), BMI $19.9-37.2 \mathrm{~kg} / \mathrm{m}^{2}$, (median $24.8 \mathrm{~kg} / \mathrm{m}^{2}$ ) in the non-pain group. A total of 15 values were missing and were imputed prior to further data analyses. DNA methylation was generally lower in TLR4 and OPRM1 than in LINE1; specifically, the median methylation across all gene- specific sites and patient subgroups were median [range], TLR4: $2.04 \%$ [0-7.5\%], OPRM1: $8.08 \%$ [0-39.25\%], LINE1: 77.78\% [65.19-100\%].

Significant differences in DNA methylation between the phenotype groups were observed for $\mathrm{CpG}$ sites in OPRM1 and in LINE1 (Fig. 1b). DNA methylation tended to be lower in the "persistent pain" phenotype group (Table 1), which agreed with the correlations between LINE1 methylation and pain ratings which were negative when statistically significant (Fig. 2). In addition, while the correlation structure (Fig. 2) indicated correlations of $\mathrm{CpG}$ site methylations within genes, among genes, and between genes and pain ratings, not every variable was correlated with the others. Interestingly, at OPRM1 CpG sites, the methylation seemed to be significantly correlated with pain ratings only when also correlated with the methylation at LINE1 CpG sites. By contrast, the methylation at TLR4 sites displayed the lowest degree of correlation with the methylation at the other genes or with pain ratings.

\section{Agreement of DNA methylation patterns with the pain- related phenotype group structure}

Swarm intelligence-based data projection followed by U-matrix visualization of the cluster structure supported DNA methylation-derived data structure that reflected the known pain phenotype group structure. Following successful swarm learning, DataBots carrying items with similar features were placed in groups on the projection grid. The distances visualized on the U-matrix indicated a large gap in the data space as a range of large so-called U-heights separating two clusters in which low Uheights indicated that the points are close to each other in the data space, indicating structure in the data set (Fig. 3a). Superimposing onto the cluster structure, the class labeling into patients with persistent or nonpersistent pain indicated a separation of the two phenotype groups by the cluster structure $\left(\chi^{2}=33.635, \mathrm{df}=1\right.$, $\left.p=6.649 \times 10^{-9}\right)$.

Results of the PC-corr analysis (see Additional file 1) supported a non-centered PCA without data transformation as adequate for further group association analyses. Specifically, although the highest amount of variance was explained by the first PC (PC1) when using centering (99.83\% versus $99.65 \%$ without centering), the noncentered analysis provided higher values of AUC and AUPR indicating slightly better group segregation. Therefore, PCA was done on the non-centered data and further analyses were applied to non-transformed data. Sample segregation along PC1 had $p$ value $<0.001$, AUC-ROC of 0.7, AUC-PR of 0.6, and explained 99.65\% of the variance. Plotting PC1 against $\mathrm{PC} 2$, which with non-centered and untransformed data explained the second largest amount of variance indicated a structure in 

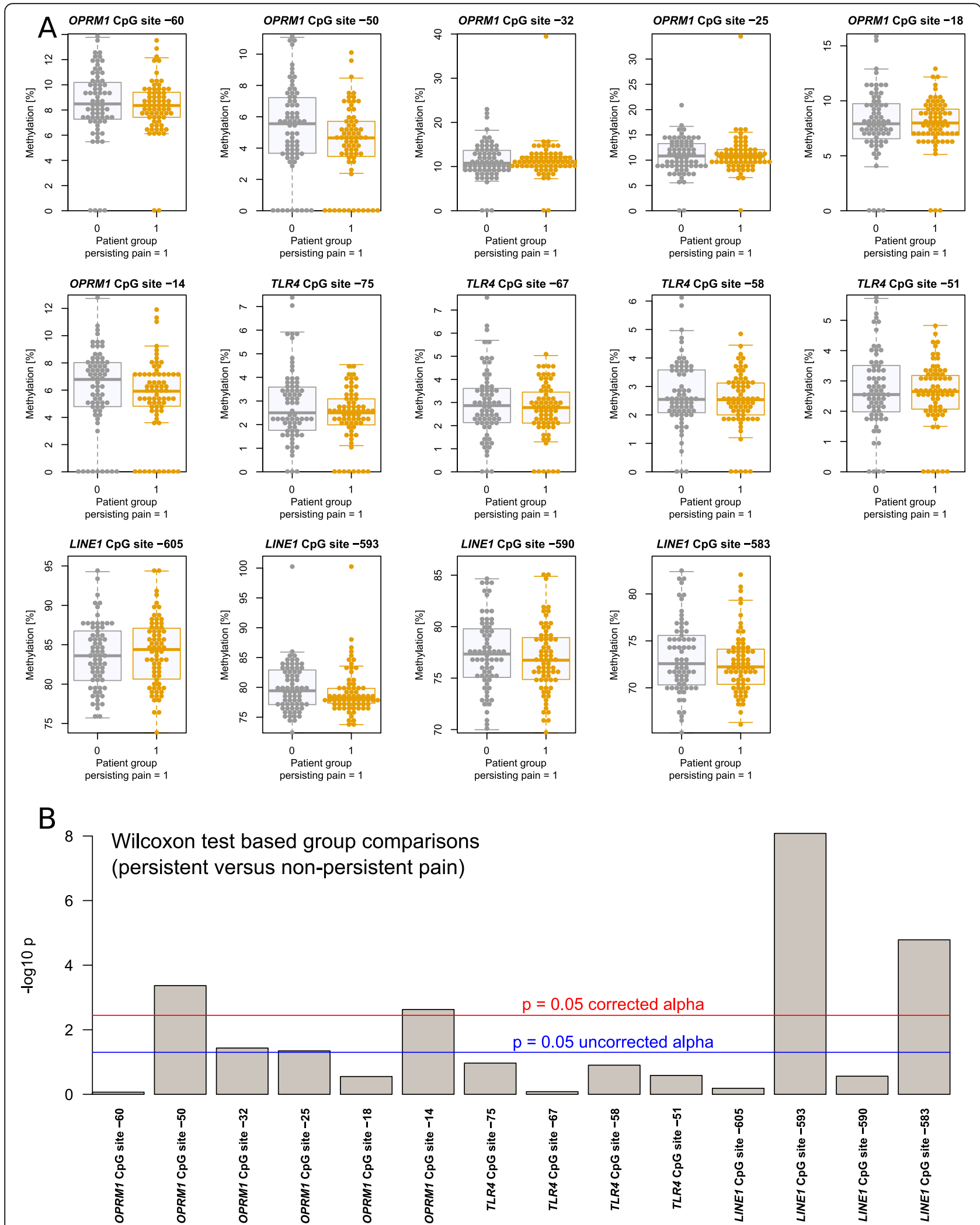

Fig. 1 (See legend on next page.) 
(See figure on previous page.)

Fig. 1 Methylation at $d=14 \mathrm{CpG}$ sites located in the OPRM1 or TLR4 genes or in the retrotransposon LINE1 (raw data, for numerical results, see also Table 1). a Raw data are shown separately for group membership to the persistent pain or non-persistent pain phenotype groups. The widths of the boxes are proportional to the respective numbers of subjects per group. The quartiles and medians (solid horizontal line within the box) are used to construct a "box and whisker" plot. The whiskers add 1.5 times the interquartile range (IQR) to the 75 th percentile or subtract 1.5 times the IQR from the 25th percentile and are expected to include $99.3 \%$ of the data if normally distributed. The notches indicate the confidence interval around the median based on median $\pm 1.57 \cdot 1 \mathrm{QR} / n^{0.5} \cdot \mathbf{b}$ Results of Wilcoxon tests for group differences in the methylation status at each CpG sites. The bars indicate the obtained $p$ values, rescaled as $-\log _{10}(p)$. Uncorrected and corrected significance thresholds are shown as horizontal lines. A significant difference is found when the bar exceeds the line. The figure has been created using the R software package (version 3.4.4 for Linux; http://CRAN.R-project.org/ [40])

the DNA methylation data that supported the separation of the two pain-related phenotype groups to which the patients had been assigned (Fig. 4).

\section{Association of specific gene methylation sites with pain phenotype group membership}

PCA of the non-centered and non-transformed data provided 11 PCs with eigenvalues exceeding the widely accepted limit of a value of 1 [66]. However, as reported above, the first component explained already > 99\% of the total variance (Table 2). It carried loadings mainly from the methylation data of $\mathrm{CpG}$ sites located in LINE1. The second PC, explaining only $0.15 \%$ of the variance, carried mainly loadings related to methylation of CpG sites in OPRM1.

Supervised machine learning algorisms trained with methylation data succeeded in pain phenotype group association to different degrees depending on the combinations of gene-related $\mathrm{CpG}$ sites used for training.

Table 1 DNA methylation observed at CpG sites in the OPRM1 and TLR4 genes and in the LINE1 retrotransposon used to quantify global DNA methylation. Means and standard deviations (SD) are given separately for the "non-persistent pain" and "persistent pain" phenotype groups

\begin{tabular}{llllll}
\hline CpG site & \multicolumn{2}{l}{ Non-persistent pain } & & \multicolumn{2}{l}{ Persistent pain } \\
\cline { 2 - 3 } \cline { 6 - 7 } & Mean & SD & & Mean & SD \\
\hline OPRM1 CpG site-60 & 8.26 & 2.76 & & 8.3 & 2.74 \\
OPRM1 CpG site-50 & 5.5 & 2.8 & & 3.83 & 2.39 \\
OPRM1 CpG site-32 & 11.95 & 5.21 & & 10.29 & 3.28 \\
OPRM1 CpG site-25 & 11.39 & 4.32 & & 10.12 & 3.17 \\
OPRM1 CpG site-18 & 8.1 & 2.71 & & 7.57 & 2.99 \\
OPRM1 CpG site-14 & 6.49 & 2.7 & & 4.93 & 3.04 \\
TLR4 CpG site-75 & 2.77 & 1.36 & & 2.47 & 1.39 \\
TLR4 CpG site-67 & 2.82 & 1.29 & & 2.82 & 1.43 \\
TLR4 CpG site-58 & 2.74 & 1.08 & & 2.49 & 1.22 \\
TLR4 CpG site-51 & 2.8 & 1.22 & & 2.51 & 1.21 \\
LINE1 CpG site-605 & 83.6 & 4.32 & & 84.07 & 4.2 \\
LINE1 CpG site-593 & 81.56 & 4.75 & & 77.56 & 1.59 \\
LINE1 CpG site-590 & 77.47 & 3.71 & & 76.74 & 3.07 \\
LINE1 CpG site-583 & 74.27 & 4.02 & 71.66 & 2.33 \\
\hline
\end{tabular}

When trained with the whole set of gene methylation information, the classification performance ranged between $71 \%$ (CART) and 80\% (SVM). In all reduced set scenarios where LINE1 methylation data were included in the training, the classification performance was similar to that obtained when training the algorithms for the complete information about DNA methylation. Moreover, when performing the training only with LINE1 methylation information, the classification performance was maintained. By contrast, while classification performance was still better than change when including OPRM1 methylation in the training data subset, it was worse than in the LINE1 containing scenarios, whereas training with TLR4 methylation provided a classification not better than chance. Indeed, hierarchical clustering of the decreases in the classification accuracy from the accuracy obtained with the full information identified two clusters for the different scenarios with respect to gene subset inclusion (Fig. 5). One cluster comprised similar classification performance as the full data set and included all scenarios where LINE1 methylation information was included. By contrast, the second cluster comprised reduced classification performances and included all scenarios without LINE1. Importantly, for all scenarios when trained with permuted methylation data, the classification performance of the algorithms was $50 \%$, i.e., like guessing (Table 3 ).

\section{Discussion}

Supporting the hypothesis of an epigenetically modulated component of persistent pain after breast cancer surgery, the degree of global methylation quantified at four CpG sites in the retrotransposon LINE1 was correlated with the pain ratings acquired from 6 to 36 months after breast cancer surgery. The agreement of the data structure emerging in the degrees of gene methylation, detected by applying unsupervised data analysis methods including machine learning with the a priori classification of the patient cohort into a "persistent" and a "non-persistent pain" phenotypic subgroup, selected as representing the extreme pain-related phenotypes from a cohort of 1000 women treated surgically for breast cancer, provided further support to the hypothesis of an epigenetically modulated component of persistent pain after breast 


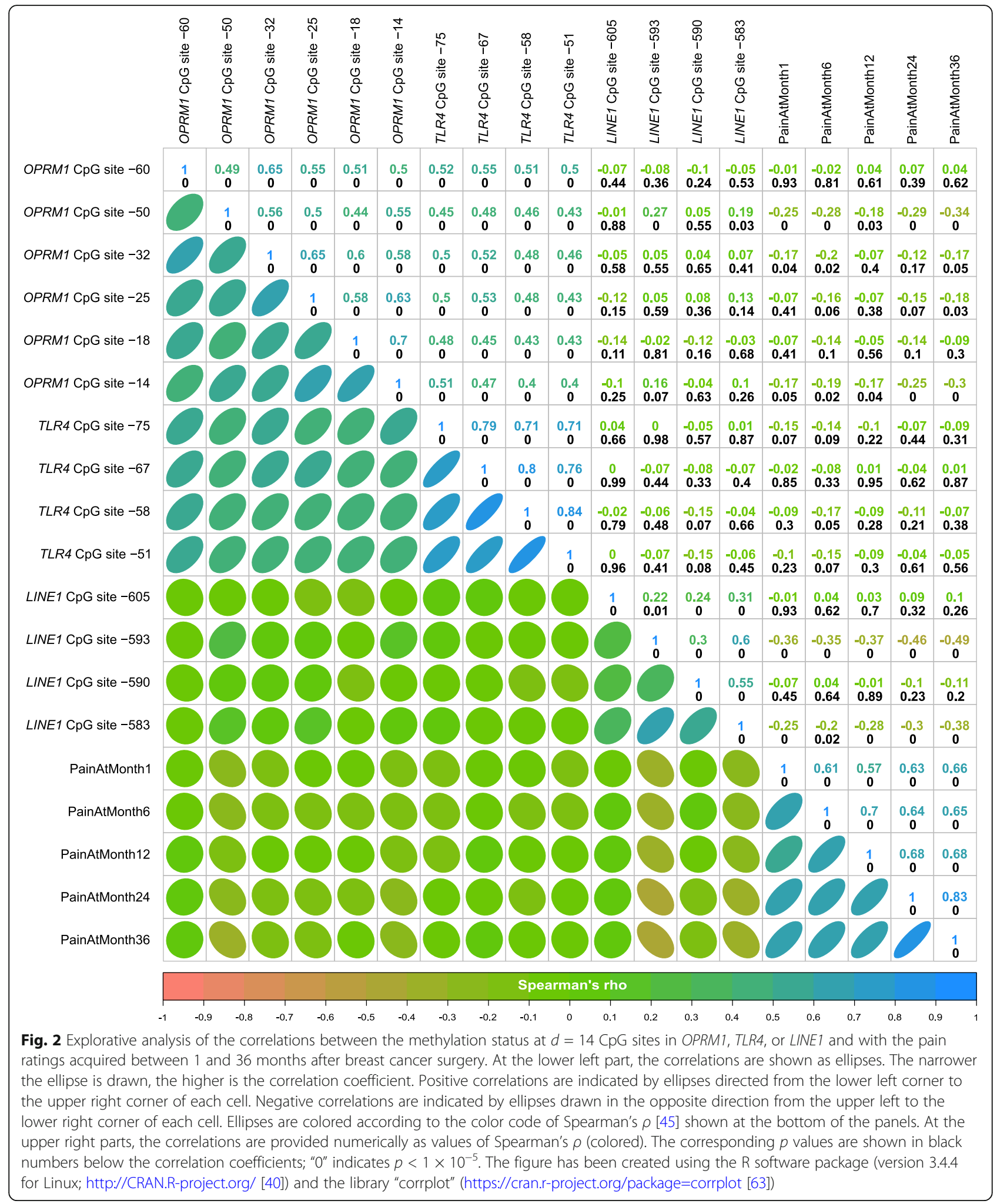

cancer surgery. Additional support for this hypothesis was provided by the ability of five different supervised algorithms, including machine learning, to assign a patient to the correct pain-phenotype subgroup based on the training with the information about the DNA methylation. This succeeded with an even higher accuracy than that obtained with a recently proposed rule-based classifier, created in the same cohort from demographic, 


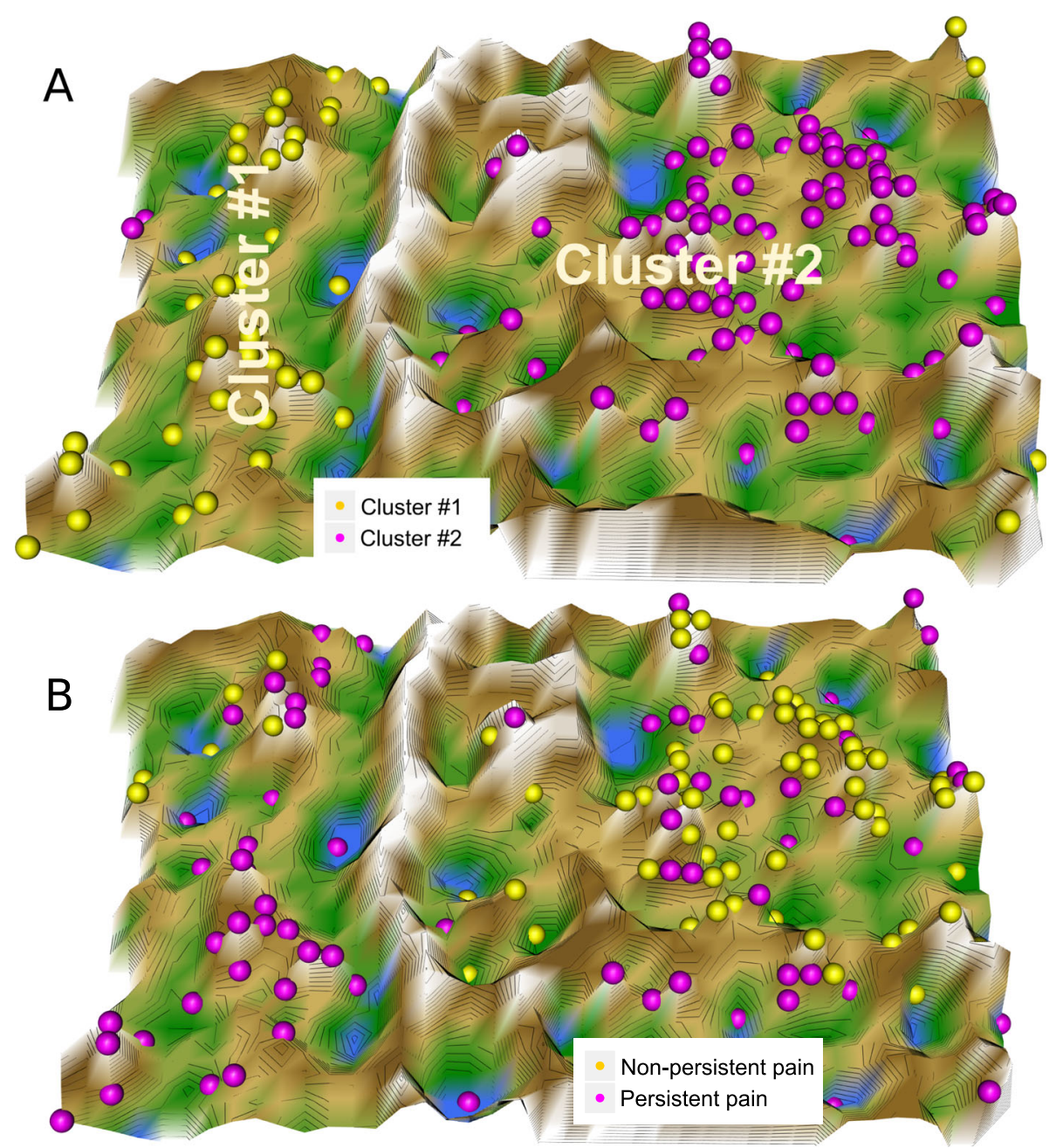

Fig. 3 Clustering of subjects based on DNA methylation at CPG sites in OPRM1, TLR4, and LINE1, obtained using unsupervised machine learning. U-matrix visualization of the data structure found via a projection onto a toroid neuronal grid using a parameter-free polar swarm, Pswarm consisting of so-called DataBots, which are self-organizing artificial "life forms" that carry vectors of the DNA methylation. a The U-matrix visualization was colored as a top view of a topographic map with brown (up to snow-covered) heights and green valleys with blue lakes. Watersheds indicate borderlines between two different clusters. b Superimposing the pain phenotype group structure indicated considerable coincidence with the cluster separation, which was supported by a significant $x^{2}$ test of the cross table of clusters versus pain phenotype groups. Please note the different meaning of the coloring of the data points in the two panels, cluster in panel $\mathbf{a}$ but pain phenotype groups in panel $\mathbf{b}$. The figure has been created using the R software package (version 3.4.4 for Linux; http://CRAN.R-project.org/ [40]) and the library "DatabionicSwarm", https://cran.r-project.org/package=DatabionicSwarm [64])

psychological, and pain-related, but not genetic or epigenetic parameters, which provided a sensitivity and specificity of 82.4 and $55.6 \%$, respectively, and an accuracy of $69 \%$ [25] to identify patients with persistent pain. Please note that the then used definition of persistent pain reports NRS $\geq 4$ as a criterion, which is the same as the presently used NRS > 3 criterion, considering that the NRS is integer-scaled. For example, the class assignment based on the epigenotypes, achieved using support vector machines (Table 3), provided a sensitivity and specificity of 69.6 and $91.3 \%$, respectively, and an accuracy of $80.4 \%$. By contrast, when training the artificial intelligence with permuted epigenetic information, its classification performance dropped to the level of guessing, supporting that the classification success was not due to overfitting. This is promising with respect to using epigenetic information as a biomarker for pain persistence. Moreover, a combination of several kinds of information, genetic, epigenetic, clinical, demographic, i.e., a combination of the previously and presently reported positive results seems a promising approach to a better biomarker, which, however, would greatly exceed the present report.

An association of the global DNA methylation status with persistent pain has previously been suggested by preclinical and clinical research. For example, in rats with post nerve injury pain, the genome-wide DNA 


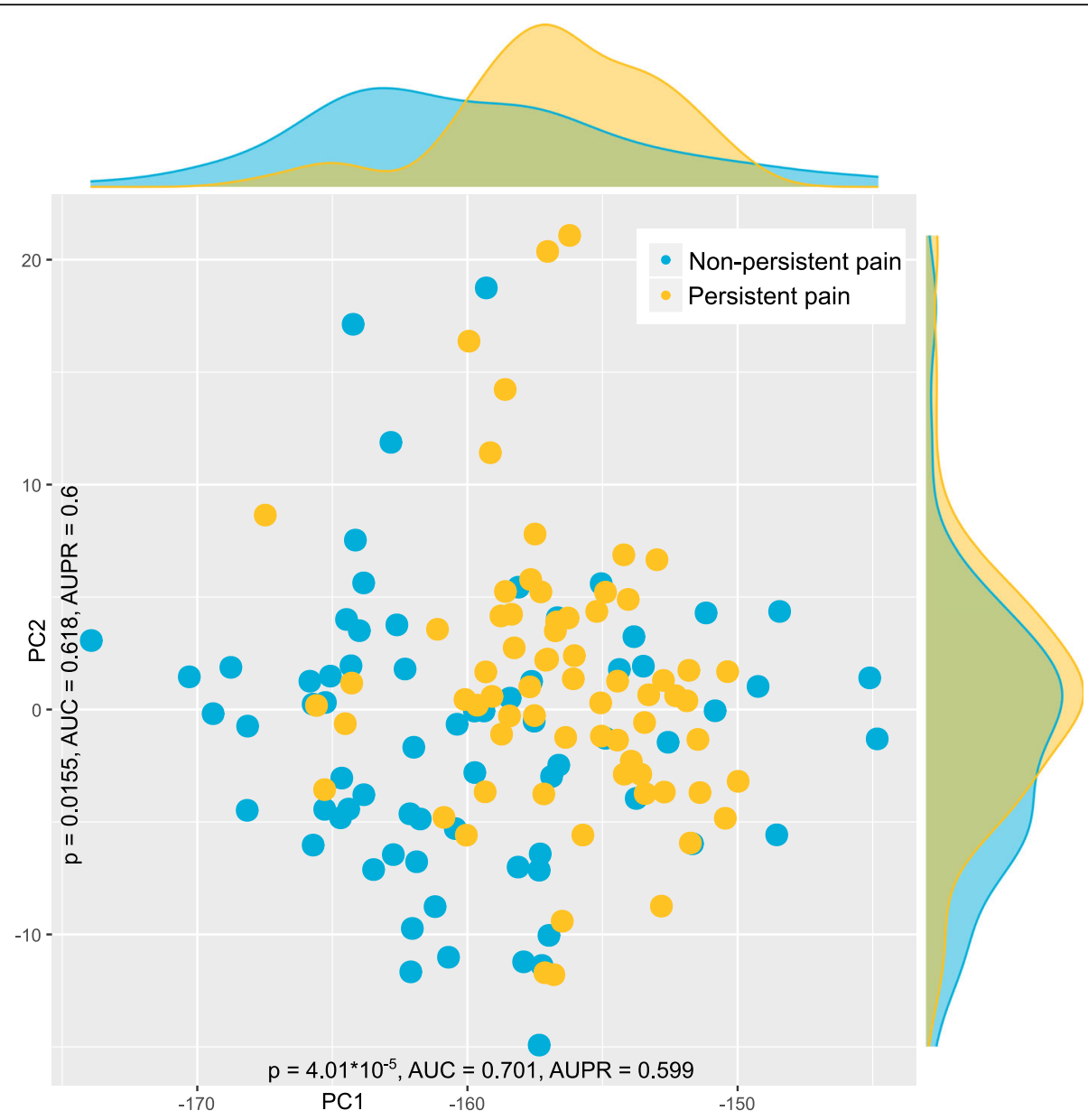

Fig. 4 Data structure found in the input space of $d=14 \mathrm{CpG}$ methylations acquired from patients with either persistent $(n=70)$ or non-persistent $(n=70)$ pain after breast cancer surgery. The data structure has been obtained by means of data projection principal component analysis on the non-normalized data as suggested by the results of the PC-corr analysis [50]. The PCA plot associated to this analysis shows the sample separation in the first and second component (PC1 versus PC2) yielded the best explained variance for non-normalized, non-centered PCA. The marginal distribution plots show the segregation of the pain phenotype groups along the first principal component. The figure has been created using the R software package (version 3.4.4 for Linux; http://CRAN.R-project.org/ [40]) and the library "ggplot2" (https://cran.r-project.org/package=ggplot2 [65])

methylation in the prefrontal cortex and in $\mathrm{T}$ cells was found to differ among pain phenotype groups, based on analysis of methylated DNA immunoprecipitation followed by hybridization to microarrays [68]. Among the all measured probes, methylation was decreased in 14,298 probes and increased in 9088 in animals with a spared nerve injury. Also in rats, nerve injury was shown to cause DNA methylation changes at $8 \%$ of the CpG sites across the whole genome, with prevailing hypomethylation outside of $\mathrm{CpG}$ islands, based on digital restriction enzyme analysis of methylation in dorsal root ganglion tissue [69]. Nerve injury caused DNA methylation changes at $8 \%$ of $\mathrm{CpG}$ sites with prevailing hypomethylation outside of $\mathrm{CpG}$ islands, in introns, intergenic regions, and repetitive sequences. However, it caused more gains of methylation in the spinal cord and prefrontal cortex. In humans, global DNA methylation differed significantly between patients with low back pain and controls, based on enzyme-linked immunosorbent assays of white blood cells [70]. Furthermore, in outpatients treated in a pain unit of tertiary care, significant positive correlation between the methylation of CpG sites located in LINE1 and pain ratings have been reported [23]. Taken together, the association of higher or lower pain with higher or lower global DNA methylation has been inconsistently reported. While in the present samples, higher methylation of LINE1 was correlated with lower pain ratings, in a previously analyzed mixed cohort of outpatients of a tertiary care pain treatment unit, the correlation had been positive [23]. In that study, a methylating effect of opioid treatment had been proposed based on higher methylation levels in opioid treated than in non-opioid treated pain patients. This effect has been reproduced independently [71]. 
Table 2 Results of the principal component analysis (PCA) performed at non-normalized and non-centered data, as suggested by the results of the PC-corr analysis [50]. Component loadings of the methylation at CpG sites are shown for PCs with eigenvalues greater than 1. Most of the variance, however, was explained already by the first principal component, PC1

\begin{tabular}{llllllllllll}
\hline Component & $P C 1$ & $P C 2$ & $P C 3$ & $P C 4$ & $P C 5$ & $P C 6$ & $P C 7$ & $P C 8$ & $P C 9$ & $P C 10$ & PC11 \\
\hline Eigenvalue & 25066.74 & 38.56 & 12.78 & 8.35 & 5.67 & 4.97 & 4.45 & 3.5 & 3.24 & 2.38 & 2.13 \\
Explained variance & 0.9966 & 0.00153 & 0.00051 & 0.00033 & 0.00023 & 0.0002 & 0.00018 & 0.00014 & 0.00013 & 0.00009 & 0.00008 \\
Cumulative variance explained & 0.9966 & 0.99808 & 0.99859 & 0.99892 & 0.99914 & 0.9993 & 0.99952 & 0.99966 & 0.99979 & 0.99988 & 0.99997 \\
OPRM1 CpG site-60 & -0.05 & -0.32 & 0.21 & 0.1 & -0.43 & -0.05 & -0.11 & 0.07 & -0.58 & -0.53 & 0.11 \\
OPRM1 CpG site-50 & -0.03 & -0.3 & -0.03 & -0.2 & -0.36 & 0.41 & 0.2 & -0.46 & 0.36 & -0.18 & -0.41 \\
OPRM1 CpG site-32 & -0.07 & -0.52 & 0.09 & 0.22 & -0.25 & -0.58 & -0.06 & -0.16 & 0.29 & 0.37 & 0.15 \\
OPRM1 CpG site-25 & -0.07 & -0.43 & -0.04 & 0.26 & 0.19 & 0.26 & 0.3 & 0.64 & 0.32 & -0.19 & 0.09 \\
OPRM1 CpG site-18 & -0.05 & -0.36 & 0.03 & -0.13 & 0.51 & -0.22 & -0.26 & 0.03 & -0.19 & -0.05 & -0.65 \\
OPRM1 CpG site-14 & -0.04 & -0.37 & -0.05 & -0.29 & 0.45 & 0.21 & -0.13 & -0.38 & -0.05 & -0.09 & 0.59 \\
TLR4 CpG site-75 & -0.02 & -0.12 & 0.06 & -0.01 & 0 & 0.22 & 0.05 & 0.01 & -0.21 & 0.39 & -0.01 \\
TLR4 CpG site-67 & -0.02 & -0.12 & 0.09 & 0.02 & -0.07 & 0.26 & 0.01 & 0.07 & -0.21 & 0.37 & -0.02 \\
TLR4 CpG site-58 & -0.02 & -0.1 & 0.08 & -0.01 & -0.08 & 0.2 & 0.05 & 0.09 & -0.22 & 0.3 & -0.08 \\
TLR4 CpG site-51 & -0.02 & -0.11 & 0.08 & -0.01 & -0.08 & 0.22 & 0.03 & 0.06 & -0.23 & 0.34 & -0.01 \\
LINE1 CpG site-605 & -0.53 & 0.17 & 0.8 & -0.07 & 0.12 & 0.02 & 0.05 & -0.03 & 0.16 & -0.04 & 0.02 \\
LINE1 CpG site-593 & -0.5 & -0.01 & -0.32 & -0.55 & -0.27 & -0.02 & -0.35 & 0.35 & 0.12 & 0.06 & 0.06 \\
LINE1 CpG site-590 & -0.49 & 0.08 & -0.28 & 0.65 & 0.06 & 0.25 & -0.37 & -0.22 & 0 & -0.02 & -0.05 \\
LINE1 CpG site-583 & -0.46 & 0.01 & -0.31 & -0.04 & 0.1 & -0.26 & 0.71 & -0.14 & -0.3 & 0 & -0.02 \\
\hline
\end{tabular}

\begin{tabular}{|c|c|c|c|c|c|c|c|c|c|c|c|}
\hline \multirow{3}{*}{ 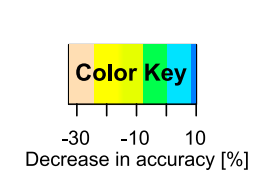 } & \multirow[b]{2}{*}{1.1} & \multirow[b]{2}{*}{2.2} & \multirow[b]{2}{*}{0} & \multirow[b]{2}{*}{2.2} & \multirow[b]{2}{*}{4.4} & \multirow{2}{*}{\multicolumn{2}{|c|}{$\begin{array}{l}\text { In the anaylsis } \\
\text { LINE } 1\end{array}$}} & & \multicolumn{3}{|c|}{ Not in the anaylsis } \\
\hline & & & & & & & & & \multicolumn{2}{|r|}{ TLR4 } & OPRM1 \\
\hline & 2.2 & 2.2 & 0 & 2.2 & 2.2 & LINE 1 & TLR4 & & & & OPRM1 \\
\hline & 0 & 0 & 0 & -2.2 & 2.2 & LINE 1 & & OPRM1 & & $T L R 4$ & \\
\hline & 0 & 0 & 0 & 0 & 0 & LINE 1 & $T L R 4$ & OPRM1 & & & \\
\hline & -21.7 & -26.1 & -32.6 & -15.2 & -21.7 & & TLR4 & & LINE 1 & & OPRM1 \\
\hline & -17.4 & -19.6 & -15.2 & -8.7 & -15.2 & & TLR4 & OPRM1 & LINE 1 & & \\
\hline & -15.2 & -17.4 & -19.6 & -13 & -15.2 & & & OPRM1 & LINE 1 & TLR4 & \\
\hline & $\frac{\sqrt[\leftarrow]{\alpha}}{\underset{\sigma}{\sigma}}$ & $\underset{z}{Z}$ & $\sum_{\infty}^{\sum}$ & 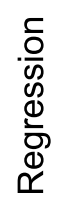 & 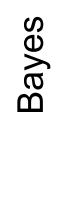 & & & & & & \\
\hline $\begin{array}{l}\text { Fig. } 5 \text { Analysis of the } \\
\text { neighbors (kNN), supr } \\
\text { methylation informati } \\
\text { The numbers indicate } \\
\text { to that obtained with } \\
\text { these differences, a p } \\
\text { reduced data set for } \\
\text { using the R software } \\
\text { Warnes; https://cran.r- }\end{array}$ & $\begin{array}{l}\text { in the cla } \\
\text { ctor mac } \\
\text { ginally c } \\
\text { ifference } \\
\text { spective } \\
\text { of two g } \\
\text { g. By con } \\
\text { ye (versio } \\
\text { t.org/pac }\end{array}$ & $\begin{array}{l}\text { fication } \\
\text { hes (SVN } \\
\text { prising } \\
\text { classific } \\
\text { gorithm } \\
\text { ups of th } \\
\text { st, the a } \\
\text { 3.4.4; htt } \\
\text { ge=gplo }\end{array}$ & $\begin{array}{l}\text { Euracy }(T \\
\text { multinon } \\
\text { btal of } d \\
\text { on accur } \\
\text { hen train } \\
\text { ested sc } \\
\text { uracy dro } \\
\text { CRAN.R- }\end{array}$ & $\begin{array}{l}\text { le 3) of } \\
\text { I regres } \\
14 \mathrm{CpG} \\
\text {, obtair } \\
\text { with th } \\
\text { arios en } \\
\text { eed in s } \\
\text { oject.or }\end{array}$ & $\begin{array}{l}\text { differe } \\
\text { ("regre } \\
\text { s locat } \\
\text { in seve } \\
\text { ill data } \\
\text { ged. In } \\
\text { arios in } \\
\text { 40]) anc }\end{array}$ & $\begin{array}{l}\text { algorithms } \\
\text { on"), and } \\
\text { in OPRM1, } \\
\text { training sc } \\
\text { Subsequ } \\
\text { first clust } \\
\text { ded in the } \\
\text { ee "heatm }\end{array}$ & $\begin{array}{l}\text { (classific } \\
\text { aïve Bay } \\
\text { TLR4, or } \\
\text { enarios c } \\
\text { ntly, app } \\
\text { r (top), t } \\
\text { second } \\
\text { p.2" func }\end{array}$ & $\begin{array}{l}\text { tion and re } \\
\text { es adaptive } \\
\text { INET, was r } \\
f \text { reduced s } \\
\text { lying hierar } \\
\text { he accuracy } \\
\text {-luster (bott } \\
\text { tion of the }\end{array}$ & $\begin{array}{l}\text { ession tree } \\
\text { ssification) } \\
\text { uced to tw } \\
\text { of gene-s! } \\
\text { cal clusteri } \\
d \text { not chan } \\
\text { ). The figu } \\
\text { backage " } g\end{array}$ & $\begin{array}{l}\text { (CART), } \\
\text { when th } \\
\text { or one } \\
\text { ecific Cp } \\
\text { g (Ward } \\
\text { ge when } \\
\text { e has be } \\
\text { lots" (G.F }\end{array}$ & $\begin{array}{l}\text { k-nearest } \\
\text { genes. } \\
\text { G islands, } \\
\text { [67]) to } \\
\text { using a } \\
\text { en created } \\
\text { ? }\end{array}$ \\
\hline
\end{tabular}


Table 3 Test performance given as accuracy in percentage for the correct assignment to the "persistent pain" patient group (upper part of the table) and as the area under the receiver operator characteristic (AUC ROC, lower part of the table), provided by different types of classifiers obtained using classification and regression trees (CART), $k$-nearest neighbors $(\mathrm{kNN})$, support vector machines (SVM), multinomial regression, and naïve Bayes adaptive classification. The first lines show the obtained accuracy when all $d=14$ CpG sites were included. Subsequently, several scenarios of reduced sets of gene-specific CpG islands were used for the training of the algorithms, and it was assessed how much leaving out a gene from the training influenced the overall classification accuracy. Furthermore, possible overfitting was accounted for by repeating the training with permuted methylation data created as negative control data sets (AUC ROC omitted). Parameter values were obtained during 1000 runs using Monte Carlo resampling from the original data set. The median of the classification accuracies obtained during the 1000 runs are shown

\begin{tabular}{|c|c|c|c|c|c|}
\hline & CART & kNN & SVM & Regression & Bayes \\
\hline \multicolumn{6}{|c|}{ Classification accuracy } \\
\hline \multicolumn{6}{|c|}{ Training with original data } \\
\hline All & 71.74 & 73.91 & 80.43 & 73.91 & 73.91 \\
\hline OPRM1 & 56.52 & 56.52 & 60.87 & 60.87 & 58.7 \\
\hline TLR4 & 47.83 & 47.83 & 47.83 & 56.52 & 50 \\
\hline LINE1 & 72.83 & 76.09 & 80.43 & 76.09 & 78.26 \\
\hline OPRM1 and TLR4 & 54.35 & 54.35 & 65.22 & 65.22 & 58.7 \\
\hline OPRM1 and LINE1 & 71.74 & 73.91 & 80.43 & 71.74 & 76.09 \\
\hline TLR4 and LINE1 & 73.91 & 76.09 & 80.43 & 76.09 & 78.26 \\
\hline \multicolumn{6}{|c|}{ Training with permuted data } \\
\hline All & 50 & 50 & 50 & 50 & 50 \\
\hline OPRM1 & 50 & 50 & 50 & 50 & 50 \\
\hline TLR4 & 50 & 50 & 50 & 50 & 50 \\
\hline LINE1 & 50 & 50 & 50 & 50 & 50 \\
\hline OPRM1 and TLR4 & 50 & 50 & 50 & 50 & 50 \\
\hline OPRM1 and LINE1 & 50 & 50 & 50 & 50 & 50 \\
\hline TLR4 and LINE1 & 50 & 50 & 50 & 50 & 50 \\
\hline \multicolumn{6}{|l|}{$\mathrm{ROC}$ areas } \\
\hline \multicolumn{6}{|c|}{ Training with original data } \\
\hline All & 79.35 & 73.91 & 82.61 & 80.72 & 80.15 \\
\hline OPRM1 & 59.78 & 53.31 & 61.81 & 69.19 & 63.94 \\
\hline TLR4 & 53.97 & 54.06 & 54.49 & 61.34 & 55.10 \\
\hline LINE1 & 79.02 & 76.09 & 84.31 & 82.23 & 79.96 \\
\hline OPRM1 and TLR4 & 58.60 & 53.07 & 67.86 & 72.40 & 61.81 \\
\hline OPRM1 and LINE1 & 79.49 & 73.91 & 81.47 & 80.15 & 81.66 \\
\hline TLR4 and LINE1 & 79.11 & 78.26 & 85.07 & 82.61 & 80.72 \\
\hline
\end{tabular}

Nevertheless, the global methylation levels of in median $80 \%$ in that study [23] agreed with the presently observed levels. In the present study, the patients had received a standardized perioperative treatment with opioids and none of the patients had been taking opioid medication either before or after surgery apart from codeine during the first postoperative week, a possible DNA methylation effect of opioids would not have been restricted to one of the subgroups and is therefore also unlikely to have caused the observed subgroup differences; neither can it explain the different direction of the correlation with pain ratings. In the present assessments, lower TLR4 methylation would have provided a plausible association with higher pain intensity; however, TLR4 methylation was not correlated with global or ORRM1 DNA methylation.

While the methylation of OPRM1 sites, as far as significantly associated with higher pain ratings, apparently provided support for epigenetic control of persistent pain after breast cancer surgery via neuro-immune crosstalk at the glial-opioid interface, supervised machine-learned analyses clearly contradicted this interpretation about the role of OPRM1 methylation versus global DNA methylation. The methylation at single genes was not needed to assign a patient to the correct pain-phenotype subgroup, hence, neither OPRM1 nor TLR4 methylation provided relevant information to train artificial intelligences to perform this phenotype group assignment. For example, the classification accuracy of support vector machines remained completely unaffected at 80.43 when omitting OPRM1 methylation, TLR4 methylation, or both, from the training, whereas it dropped by $15 \%$ or more when omitting LINE1 methylation (Table 3). Thus, the partly correlated methylations in OPRM1 probably just followed the global DNA methylation status reflected in LINE1, without indication that they represented a gene-specific mechanism of the regulation of postoperative persistent pain.

The interpretation that the observed epigenetic associations with the development of persistent pain after breast cancer surgery have to be attributed to the global methylation not reflecting a specific regulation in OPRM1 is unlikely to change if more than six CpG sites in OPRM1 were analyzed. Reanalyzing previously published data [23] of the methylation at 22 CpG sites in OPRM1 indicated that DNA methylation was highly positively correlated among all 22 sites, with a median value of Spearman's $\rho$ of 0.552 (range $\rho=0.256-0.747$ ) and a median significance level of $p=6.11 \times 10^{-15}$ (range $p=7.34 \times 10^{-47}-0.00077$ ). Hence, it seems unlikely that the analysis of more sites within OPRM1 would have changed the present results. However, an epigenetic control via OPRM1 or TLR4 remains possible via histone modulation, which was not assessed in the present study. It is known to play a role in pain-related human phenotypes such as the bladder pain syndrome [72] or as one of the mechanism via which valproate is effective in the management of diabetic neuropathy [73]. 
Furthermore, since DNA methylation is tissue specific [74], a negative result obtained in DNA extracted from blood cells does not exclude an epigenetic modulation via TLR4 or OPRM1 in the central nervous system. Finally, the hypothesized epigenetic control of neuroimmune crosstalk in persistent pain remains a possibility via further genes involved in the glial-opioid interface [17].

Using a case-control approach and drawing from the patients belonging to the non-persistent pain group, a similarly sized sample as the subgroup of patients with persistent pain introduced some limitations in the analysis via oversampling of cases versus controls. Specifically, the present sample was taken to compare the epigenotypes of the subjects with the extreme pain phenotypes of interest, while intermediate phenotypes were omitted. This is a standard design which has several variants such as using matched pairs. Therefore, the numerical values of the pain phenotype group association accuracy may require revision when applied to a non-selected cohort. Hence, the reported classification performances of the algorithms are not presented as a proposal of a diagnostic tool but have been used in a knowledge-discovery manner, aimed at identifying DNA locations within preselected genes where the degree of methylation is distinctive between extreme pain phenotypes after breast cancer surgery. In addition, in a larger sample, more cases available for algorithm training may lead to improved classification performance. However, while machine-learning often unveils its power in so-called big data, its definition is purely methodological as it is referred to as a set of methods that can automatically detect patterns in data and then use the uncovered patterns to predict or classify future data, to observe structures such as subgroups in the data or to extract information from the data suitable to derive new knowledge [75-77]. This meets exactly the present application of machine learning.

The limited, hypothesis-driven selection of two groups of genes, rather than performing a more comprehensive quantification of genome-wide DNA methylation in many other genes relevant to pain and its persistence, further emphasizes the knowledgediscovery focus of the present analysis. The present analysis was performed in a collaborative EU project about persistent pain and it explicitly set the "focus on glial-opioid receptor interface" (project " $D$ " in Table 1 in [78]). The two gene families were exclusively named in the project reported here. More comprehensive assessments of the role of DNA methylation in persistent pain may use other candidate gene approaches in the future. At least 540 genes have been so far demonstrated to be relevant to pain [79-82]. Those approaches may also address the DNA methylation across the whole genome without a restriction to prior knowledge about painrelevant sites.

\section{Conclusions}

Using information on the methylation of CpG sites, machine-learned analysis indicated that the epigenotypes provide useful information for the allocation of the patients to either a "persistent pain" or "non-persistent pain" phenotype group in a 3-year follow-up after breast cancer surgery. The global DNA methylation, quantified at CpG sites located in the retrotransposon LINE1, provided a similar diagnostic accuracy for persistent pain as the previously established non-genetic predictors, based on a single DNA sample. By contrast, the methylation of TLR4 or OPRM1 genes could not contribute further to the allocation of the patients to the pain-related phenotype groups. Therefore, the present analysis cannot provide support for specific epigenetic modulation of persistent postoperative pain via methylation of two key genes of the glial-opioid interface. Finally, although the findings regarding the focused hypothesis of a regulation of persistent pain via methylation of OPRM1 and TLR4 genes were negative, an accuracy of group assignment approaching $80 \%$ by using global epigenetic information encourages further exploration of DNA methylation as a possibly important component of a future biomarker for risk of persistent pain. The analysis emphasizes the need to include a marker for global DNA methylation in epigenetic analyses to prevent that an effect, such as a group difference, being wrongly attributed to the methylation of a specific gene.

\section{Supplementary information}

Supplementary information accompanies this paper at https://doi.org/10 1186/s13148-019-0772-4.

Additional file 1. Supplemental information includes a table of results of a PC-corr analysis of the data in Microsoft Excel format (PCcorr_results.xIsx).

\section{Abbreviations}

DNA: Deoxyribonucleic acid; LINE1: LINE1 retrotransposable element 1; NRS: Numeric rating scale; OPRM1: Opioid receptor mu 1; PCA: Principal component analysis; TLR4: Toll-like receptor 4

\section{Acknowledgements}

Clinical but not epigenetic data derived from this study have been analyzed in a non-redundant manner published in Breast Cancer Res Treat. 2018 Jun 6. doi: 10.1007/s10549-018-4841-8, Br J Anaesth 2018. 2018 Nov;121(5):11231132, Br J Anaesth. 2017 Oct 1;119(4):821-829, J Clin Oncol 2017; 35: 16601667, and Pain 2019 May 15. doi: 10.1097/j.pain.0000000000001616.

\section{Authors' contributions}

$E K, J \mathrm{~L}$, and DK conceived and designed the experiments. EK and MAK performed the clinical data acquisition. DK performed the epigenetic analyses. $J \mathrm{~L}$ analyzed the data. $J$, DK, EK, and MAK wrote the paper. $J \mathrm{~L}$ and EK revised the paper. All authors read and approved the final manuscript.

\section{Funding}

The work has been supported by the European Union Seventh Framework Programme (FP7/2007-2013) under grant agreement no. 602919 (GLORIA, EK, JL) by the Academy of Finland (EK), by the Helsinki University Hospital Governmental Research funds (TYH2008225, TYH2010210, EK), and by the 
Landesoffensive zur Entwicklung wissenschaftlich-ökonomischer Exzellenz (LOEWE), LOEWE-Zentrum für Translationale Medizin und Pharmakologie (JL). The funders had no role in method design, data selection and analysis, decision to publish, or preparation of the manuscript.

\section{Availability of data and materials}

The patients' consent does not include public availability of source data or materials.

\section{Ethics approval and consent to participate}

The study followed the Declaration of Helsinki and both the Coordinating Ethics Committee (journal number 136/E6/2006) and the Ethics Committee of the Department of Surgery (148/E6/05) of the Hospital District of Helsink and Uusimaa approved the study protocol. Informed written consent was obtained from each patient.

\section{Consent for publication}

Informed written consent was obtained from all subjects where they agreed to an anonymous analysis and publication of the findings.

\section{Competing interests}

The authors declare that they have no competing interests.

\section{Author details}

'Institute of Clinical Pharmacology, Goethe-University, Theodor-Stern-Kai 7, 60590 Frankfurt am Main, Germany. ${ }^{2}$ Division of Pain Medicine, Department of Anesthesiology, Intensive Care and Pain Medicine, University of Helsinki and Helsinki University Hospital, Helsinki, Finland. ${ }^{3}$ Fraunhofer Institute of Molecular Biology and Applied Ecology-Project Group Translational Medicine and Pharmacology (IME-TMP), Theodor-Stern-Kai 7, 60590 Frankfurt am Main, Germany. ${ }^{4}$ Institute for Molecular Medicine Finland (FIMM), HiLIFE, University of Helsinki, Helsinki, Finland.

Received: 22 May 2019 Accepted: 23 October 2019

Published online: 27 November 2019

\section{References}

1. Liu T, Gao YJ, Ji RR. Emerging role of Toll-like receptors in the control of pain and itch. Neurosci Bull. 2012;28(2):131-44.

2. Landmark T, Dale O, Romundstad P, Woodhouse A, Kaasa S, Borchgrevink PC. Development and course of chronic pain over 4 years in the general population: The HUNT pain study. Eur J Pain. 2018;22(9):1606-16.

3. Woolf CJ, Salter MW. Neuronal plasticity: increasing the gain in pain. Science. 2000;288(5472):1765-9.

4. Stucky CL, Gold MS, Zhang X. Mechanisms of pain. Proc Natl Acad Sci U S A. 2001:98(21):11845-6.

5. Ji RR, Kohno T, Moore KA, Woolf CJ. Central sensitization and LTP: do pain and memory share similar mechanisms? Trends Neurosci. 2003: 26(12):696-705.

6. Li HL, Qin LY, Wan Y. Astrocyte: a new star in pain research. Sheng Li Ke Xue Jin Zhan. 2003;34(1):45-8.

7. Scholz J, Woolf CJ. The neuropathic pain triad: neurons, immune cells and glia. Nat Neurosci. 2007;10(11):1361-8.

8. Gao YJ, Ji RR. Chemokines, neuronal-glial interactions, and central processing of neuropathic pain. Pharmacol Ther. 2010;126(1):56-68.

9. Lacagnina MJ, Watkins LR, Grace PM. Toll-like receptors and their role in persistent pain. Pharmacol Ther. 2018;184:145-58.

10. Yao L, Kan EM, Lu J, Hao A, Dheen ST, Kaur C, et al. Toll-like receptor 4 mediates microglial activation and production of inflammatory mediators in neonatal rat brain following hypoxia: role of TLR4 in hypoxic microglia. J Neuroinflammation. 2013;10:23.

11. Hutchinson MR, Zhang Y, Brown K, Coats BD, Shridhar M, Sholar PW, et al. Non-stereoselective reversal of neuropathic pain by naloxone and naltrexone: involvement of toll-like receptor 4 (TLR4). Eur J Neurosci. 2008; 28(1):20-9.

12. Tian L, Ma L, Kaarela T, Li Z. Neuroimmune crosstalk in the central nervous system and its significance for neurological diseases. I Neuroinflammation. 2012;9:155.

13. Fan YX, Hu L, Zhu SH, Han Y, Liu WT, Yang YJ, et al. Paeoniflorin attenuates postoperative pain by suppressing matrix metalloproteinase-9/2 in mice. Eur J Pain. 2018;22(2):272-81.
14. Chen S, Hui H, Zhang D, Xue Y. The combination of morphine and minocycline may be a good treatment for intractable post-herpetic neuralgia. Med Hypotheses. 2010;75(6):663-5.

15. Boue J, Blanpied C, Djata-Cabral M, Pelletier L, Vergnolle N, Dietrich G, Immune conditions associated with CD4+ T effector-induced opioid release and analgesia. Pain. 2012;153(2):485-93.

16. Lilius TO, Rauhala PV, Kambur O, Kalso EA. Modulation of morphine-induced antinociception in acute and chronic opioid treatment by ibudilast. Anesthesiology. 2009;111(6):1356-64.

17. Kringel D, Lippmann C, Parnham MJ, Kalso E, Ultsch A, Lotsch J. A machinelearned analysis of human gene polymorphisms modulating persisting pain points to major roles of neuroimmune processes. Eur J Pain. 2018;22(10):1735-56.

18. Doehring A, Geisslinger $G$, Lötsch J. Epigenetics in pain and analgesia: an imminent research field. Eur J Pain. 2011;15(1):11-6.

19. Chidambaran V, Zhang X, Martin LJ, Ding L, Weirauch MT, Geisler K, et al. DNA methylation at the mu-1 opioid receptor gene (OPRM1) promoter predicts preoperative, acute, and chronic postsurgical pain after spine fusion. Pharmgenomics Pers Med. 2017;10:157-68.

20. Kazazian $\mathrm{HH} \mathrm{Jr}$, Goodier JL. LINE drive, retrotransposition and genome instability. Cell. 2002;110(3):277-80.

21. Ehrlich M. DNA methylation in cancer: too much, but also too little. Oncogene. 2002;21(35):5400-13.

22. Deininger PL, Moran JV, Batzer MA, Kazazian HH Jr. Mobile elements and mammalian genome evolution. Curr Opin Genet Dev. 2003;13(6):651-8.

23. Doehring A, Oertel BG, Sittl R, Lötsch J. Chronic opioid use is associated with increased DNA methylation correlating with increased clinical pain Pain. 2013;154(1):15-23.

24. Kaunisto MA, Jokela R, Tallgren M, Kambur O, Tikkanen E, Tasmuth T, et al. Pain in 1,000 women treated for breast cancer: a prospective study of pain sensitivity and postoperative pain. Anesthesiology. 2013;119(6):1410-21.

25. Lötsch J, Sipilä R, Tasmuth T, Kringel D, Estlander AM, Meretoja T, et al. Machine-learning-derived classifier predicts absence of persistent pain after breast cancer surgery with high accuracy. Breast Cancer Res Treatment. 2018(accepted).

26. Kringel D, Geisslinger G, Resch E, Oertel BG, Thrun MC, Heinemann S, et al. Machine-learned analysis of the association of next-generation sequencing based human TRPV1 and TRPA1 genotypes with the sensitivity to heat stimuli and topically applied capsaicin. Pain. 2018(accepted).

27. Meretoja TJ, Leidenius MH, Tasmuth T, Sipila R, Kalso E. Pain at 12 months after surgery for breast cancer. JAMA. 2014;311(1):90-2.

28. Trimble EL, Ungerleider RS, Abrams JA, Kaplan RS, Feigal EG, Smith MA, et al. Neoadjuvant therapy in cancer treatment. Cancer. 1993;72(11 Suppl):3515-24.

29. Lötsch J, Sipilä R, Dimova V, Kalso E. Machine-learned selection of psychological questionnaire items relevant to the development of persistent pain after breast cancer surgery. Br J Anaesth. 2018(accepted).

30. Lötsch J, Ultsch A, Kalso E. Prediction of persistent post-surgery pain by preoperative cold pain sensitivity: biomarker development with machinelearning-derived analysis. Br J Anaesth. 2017:119(4):821-9.

31. Gagliese L, Weizblit N, Ellis W, Chan WW. The measurement of postoperative pain: a comparison of intensity scales in younger and older surgical patients. Pain. 2005;117(3):412-20.

32. Sipilä R, Estlander A-M, Tasmuth T, Kataja M, Kalso E. Development of a screening instrument for risk factors of persistent pain after breast cancer surgery. Br J Cancer. 2012:107(9):1459-66.

33. Macrae WA. Chronic pain after surgery. Br J Anaesth. 2001;87(1):88-98

34. Oertel BG, Doehring A, Roskam B, Kettner M, Hackmann N, Ferreiros N, et al. Genetic-epigenetic interaction modulates mu-opioid receptor regulation. Hum Mol Genet. 2012;21(21):4751-60

35. Knothe C, Doehring A, Ultsch A, Lötsch J. Methadone induces hypermethylation of human DNA. Epigenomics. 2016;8(2):167-79. https:/ doi.org/10.2217/epi.15.78. Epub 2015 Sep 4.

36. Bollati V, Baccarelli A, Hou L, Bonzini M, Fustinoni S, Cavallo D, et al. Changes in DNA methylation patterns in subjects exposed to low-dose benzene. Cancer Res. 2007;67(3):876-80

37. Kile ML, Baccarelli A, Tarantini L, Hoffman E, Wright RO, Christiani DC Correlation of global and gene-specific DNA methylation in maternal-infant pairs. PLoS One. 2010:5(10):e13730.

38. Florea AM. DNA methylation pyrosequencing assay is applicable for the assessment of epigenetic active environmental or clinical relevant chemicals. Biomed Res Int. 2013;2013:486072. 
39. Feraritra R, Sulistyonigrum D, Huriyati E, Sadewa A, Rinasusilowati R. Correlation of methylation of toll-like receptor 4 (TLR4) and interleukin-6 (IL6) promoter with insulin resistance in obese adolescents. J Med Sci. 2016; 48(1):11-25. https://doi.org/10.19106/JMedSci004801201602.

40. R Development Core Team. R: A Language and Environment for Statistical Computing. 2008

41. Grubbs FE. Sample criteria for testing outlying observations. Ann Math Statist. 1950;21(1):27-58.

42. Komsta L. outliers: Tests for outliers; 2011.

43. Torgo L. Data mining with R: learning with case studies: Chapman I\& Hall/ CRC; 2010. p. 305

44. Bonferroni CE. Teoria statistica delle classi e calcolo delle probabilita. Pubblicazioni del R Istituto Superiore di Scienze Economiche e Commerciali di Firenze. 1936;8:3-62

45. Spearman C. The proof and measurement of association between two things. Am J Psychol. 1904;15:72-101.

46. Ultsch A, Sieman HP. Kohonen's self organizing feature maps for exploratory data analysis. INNC'90, Int Neural Network Conference; 19901990. Dordrecht: Kluwer; 1990.

47. Lötsch J, Ultsch A. Exploiting the structures of the U-matrix. In: Villmann T, Schleif F-M, Kaden M, Lange M, editors. Advances in Intelligent Systems and Computing. 295. Heidelberg: Springer; 2014. p. 248-57.

48. Thrun MC. Projection-based clustering through self-organization and swarm intelligence: combining cluster analysis with the visualization of highdimensional data: Springer Fachmedien Wiesbaden; 2018.

49. Pearson KLIII. On lines and planes of closest fit to systems of points in space. The London, Edinburgh, and Dublin Philosophical Magazine and Journal of Science. 1901;2(11):559-72.

50. Ciucci S, Ge Y, Duran C, Palladini A, Jimenez-Jimenez V, Martinez-Sanchez $L M$, et al. Enlightening discriminative network functional modules behind principal component analysis separation in differential-omic science studies. Sci Rep. 2017;7:43946.

51. Breimann L, Friedman JH, Olshen RA, Stone CJ. Classification and regression trees. Boca Raton: Chapman and Hall; 1993.

52. Cover T, Hart P. Nearest neighbor pattern classification. IEEE Trans Inf Theor. 1967;13(1):21-7.

53. Cortes C, Vapnik V. Support-Vector Networks. Machine Learning. 1995;20(3): 273-97.

54. Walker SH, Duncan DB. Estimation of the probability of an event as a function of several independent variables. Biometrika. 1967:54(1/2):167-79.

55. Bayes M, Price M. An Essay towards solving a problem in the doctrine of chances. By the Late Rev. Mr. Bayes, F. R. S. Communicated by Mr. Price, in a Letter to John Canton, A. M. F. R. S. Philosophical Transactions. 1763:53:370-418.

56. Loh W-Y. Fifty years of classification and regression trees. International Statistical Review. 2014;82(3):329-48.

57. Karatzoglou A, Smola A, Hornik K, Zeileis A. kernlab - An S4 Package for Kernel Methods in R. Journal of Statistical Software. 2004;11(9):1-20.

58. Cox DR. Some procedures associated with the logistic qualitative response curve. New York: John Wiley \& Sons; 1966.

59. Venables WN, Ripley BD. Modern Applied Statistics with S. New York: Springer; 2002

60. Weihs C, Ligges U, Luebke K, Raabe N. klaR Analyzing German Business Cycles. Data Analysis and Decision Support. Berlin: Springer-Verlag; 2005. p. 335-43.

61. Good PI. Resampling methods : a practical guide to data analysis. Boston: Birkhäuser; 2006.

62. Tillé Y, Matei A. sampling: Survey Sampling; 2016

63. Wei T, Simko V. R package "corrplot": visualization of a correlation matrix; 2017.

64 Thrun M. DatabionicSwarm; 2017.

65. Wickham H. ggplot2: Elegant graphics for data analysis. New York: SpringerVerlag; 2009

66. Kaiser HF, Dickman K. Analytic determination of common factors. Am Psychol. 1959:14:425.

67. Ward JH Jr. Hierarchical grouping to optimize an objective function. Journal of the American statistical association. 1963;58(301):236-44.

68. Massart R, Dymov S, Millecamps M, Suderman M, Gregoire S, Koenigs K, et al. Overlapping signatures of chronic pain in the DNA methylation landscape of prefrontal cortex and peripheral T cells. Sci Rep. 2016;6:19615.
69. Garriga J, Laumet G, Chen SR, Zhang Y, Madzo J, Issa JJ, et al. Nerve injuryinduced chronic pain is associated with persistent DNA methylation reprogramming in dorsal root ganglion. J Neurosci. 2018;38(27):6090-101.

70. Kronman C, Youssef A, Murali FMV, Borsook D, Simons L. Neural response to fear learning in pediatric chronic pain. The Journal of Pain. 2018;19(3):S104.

71. Viet CT, Dang D, Aouizerat BE, Miaskowski C, Ye Y, Viet DT, et al. OPRM1 Methylation contributes to opioid tolerance in cancer patients. J Pain. 2017; 18(9):1046-59.

72. Elgavish A. Epigenetic reprogramming: a possible etiological factor in bladder pain syndrome/interstitial cystitis? J Urol. 2009;181(3):980-4.

73. Agrawal RP, Goswami J, Jain S, Kochar DK. Management of diabetic neuropathy by sodium valproate and glyceryl trinitrate spray: a prospective double-blind randomized placebo-controlled study. Diabetes Res Clin Pract. 2009;83(3):371-8.

74. Knothe C, Shiratori $H$, Resch E, Ultsch A, Geisslinger G, Doehring A, et al. Disagreement between two common biomarkers of global DNA methylation. Clin Epigenetics. 2016;8:60

75. Murphy KP. Machine learning: a probabilistic perspective: The MIT Press; 2012. p. 1096

76. Dhar V. Data science and prediction. Commun ACM. 2013:56(12):64-73.

77. Lotsch J, Ultsch A. Machine learning in pain research. Pain. 2017;159(4):623-30.

78. Kringel D, Lötsch J. Pain research funding by the European Union Seventh Framework Programme. Eur J Pain. 2015;19(5):595-600

79. Kringel D, Kaunisto MA, Lippmann C, Kalso E, Lötsch J. Development of an AmpliSeq ${ }^{\mathrm{TM}}$ panel for next-generation sequencing of a set of genetic predictors of persisting pain. Front Pharmacol. 2018(in press).

80. Lippmann C, Ultsch A, Lotsch J. Computational functional genomics-based reduction of disease-related gene sets to their key components. Bioinformatics. 2019;35(14):2362-2370. https://doi.org/10.1093/ bioinformatics/bty986

81. Lötsch J, Doehring A, Mogil JS, Arndt T, Geisslinger G, Ultsch A. Functional genomics of pain in analgesic drug development and therapy. Pharmacol Ther. 2013:139(1):60-70.

82. Ultsch A, Kringel D, Kalso E, Mogil JS, Lötsch J. A data science approach to candidate gene selection of pain regarded as a process of learning and neural plasticity. Pain. 2016;157(12):2747-57.

\section{Publisher's Note}

Springer Nature remains neutral with regard to jurisdictional claims in published maps and institutional affiliations.
Ready to submit your research? Choose BMC and benefit from:

- fast, convenient online submission

- thorough peer review by experienced researchers in your field

- rapid publication on acceptance

- support for research data, including large and complex data types

- gold Open Access which fosters wider collaboration and increased citations

- maximum visibility for your research: over $100 \mathrm{M}$ website views per year

At BMC, research is always in progress.

Learn more biomedcentral.com/submission 